\section{AFECTIVIDAD Y PROMOCIÓN DE LA SALUD EN LA ESCUELA: CONSTRUCCIÓN DE SIGNIFICADOS POR EL MAESTRO}

\author{
Afetividade e promoção da saúde na escola: construção de \\ significados para o professor
}
Affectivity and health promotion at school: the construction of meanings by the teacher

\begin{abstract}
RESUMEN
Objetivo: Comprender la afectividad y la promoción de la salud en la escuela a través de la construcción de significados por el maestro. Métodos: Estudio exploratorio y descriptivo con un enfoque cualitativo utilizando como instrumento de recolección de datos los mapas afectivos y entrevistas. Los mapas afectivos proporcionan la superación de la dualidad entre cognición, afecto y la representación de los espacios, pues en este contexto cualquier ambiente puede ser percibido como un territorio emocional. En las entrevistas se investigó las acciones promotoras de salud en las que cada maestra participó, su percepción sobre las mismas, los afectos cuando realizan las acciones sugeridas por la escuela y cómo perciben su participación en estas acciones. Resultados: Los mapas afectivos señalaron diversos y reveladores afectos de las maestras al hablar de su relación con la escuela, entre ellos, amistad, compañerismo y unidad, que se consideran importantes para que la escuela sea vista como un lugar placentero. Además, en la escuela estudiada se muestran evidentes conflictos en la relación del profesorado con los estudiantes en prácticas que realizan acciones de salud en el lugar, pues ellos no consideran al maestro ni a la comunidad educativa y esto hace emerger en los profesores un sentimiento de exclusión y desvaluación. Conclusión: Los sentimientos placenteros por el espacio escolar no son suficientes para el envolvimiento del maestro en las acciones promotoras de salud por la manera descontextualizada que son hechas, esto representa un obstáculo para la participación de los mismos.
\end{abstract}

Descriptores: Afecto; Promoción de la Salud; Educación en Salud; Docentes; Instituciones Académicas.

\section{RESUMO}

Objetivo: Compreender a afetividade e a promoção da saúde na escola através da construção de significado pelo professor. Métodos: $O$ presente estudo exploratório e descritivo foi realizado com um enfoque qualitativo utilizando como instrumento de coleta de dados os mapas afetivos e entrevistas semiestruturadas. Os mapas afetivos fornecem a superação da dualidade entre cognição, afeto e representação do espaço, pois, neste contexto, qualquer ambiente pode ser percebido como um território emocional. As entrevistas objetivaram investigar quais ações promotoras de saúde cada professor participou, sua percepção sobre elas, quais afetos emergem quando realizam as ações sugeridas pela escola e como percebem sua participação nestas ações. Os dados foram analisados a partir da análise de conteúdo, emergindo categorias temáticas. Resultados: A partir dos dados dos mapas afetivos surgiram diversos e reveladores sentimentos das professoras ao falar de sua relação com a escola entre eles a amizade, companheirismo e união. Afetos estes considerados como importantes para que a escola seja vista como um lugar prazeroso. Contudo, perceberam-se evidentes conflitos na relação dos professores com os estagiários dos cursos de graduação em saúde responsáveis pela realização das ações voltadas à saúde na escola. Eles não consideram o professor e nem a comunidade escolar e isso faz emergir sentimentos de exclusão $e$ desvalorização. Conclusão: Os sentimentos prazerosos pelo ambiente escolar não são suficientes para o envolvimento do professor nas ações promotoras de saúde pela maneira
Artigo Original
1) Universidade de Fortaleza - UNIFOR Fortaleza (CE) - Brasil

2) Universidade de Valencia - UV- Valencia - Espanha
Recebido em: 04/03/2016 Revisado em: 13/05/2016 Aceito em: 20/08/2016 
descontextualizada como estas são realizadas. O que representou um obstáculo para a participação dos docentes. Concluimos este estudio defendiendo el aumento de las discusiones sobre lo papel de los maestros en las acciones de promoción de la salud en la escuela.

Descritores: Afeto; Promoção da Saúde; Educação para a Saúde; Docentes; Instituições Acadêmicas.

\section{ABSTRACT}

Objective: To understand affectivity and health promotion at school through the construction of meanings by the teachers. Methods: The present exploratory and descriptive study was conducted based on a qualitative approach using affective maps and semi-structured interviews as tools for data collection. Affective maps transcend the duality between cognition, affect and spatial representations because, within this context, any environment can be seen as an emotional territory. The interviews aimed to investigate which health promoting actions each teacher has participated in, their perceptions regarding them, which affects emerge when carrying out the actions suggested by the school and how they perceive their participation in such actions. Results: Data from the affective maps showed several and revelatory feelings among teachers when talking about their relationship with the school, including friendship, companionship and union. These feelings are considered important to help the school be seen as a pleasurable space. However, there are evident conflicts in the relationship between teachers and the health undergraduate students who are responsible for carrying out the health promotion actions at the school. They do not take into consideration the teacher and the school community, which gives rise to feelings of exclusion and undervaluation. Conclusion: Pleasure feelings about the school environment are not enough for making teachers participate in health promoting actions due to the decontextualized manner they have been carried out. This represented an obstacle to teachers' participation. We conclude this study advocating in favor of a better discussion of the teachers' role in health promoting actions at the school.

Descriptors: Affect; Health Promotion; Health Education; Faculty; School.

\section{INTRODUCCIÓN}

La Promoción de la Salud ${ }^{(1)}$ es el proceso que permite a las personas incrementar y mejorar el control sobre su salud y sobre los factores que la determinan. Es una función básica de la salud pública y contribuye para la lucha contra las enfermedades transmisibles y no transmisibles y otras amenazas para la salud. Las intervenciones y las prácticas que tienen el objetivo de promover la salud deben estimular el análisis crítico y reflexivo sobre los valores, conductas, condiciones sociales y ambientales y los estilos de vida de la población, tratando de fortalecer lo que contribuye a mejorar la salud y la calidad del medio ambiente.

Entre los cinco tipos de acciones propuestas por la Carta de Otawa ${ }^{(1)}$ para la Promoción de la Salud, destacase el desarrollo de habilidades personales y de autonomía de los individuos. Para alcanzar y entender estos objetivos se ha ido construyendo un marco teórico y metodológico, donde tiene sentido esta investigación en la cual se destaca:

La educación para la salud se considera un importante instrumento de la Promoción de la Salud y la entendemos como el conjunto de estrategias de enseñanza-aprendizaje de carácter participativo y emancipatório que faculta a las personas a analizar e intervenir en su entorno, desarrollar las actitudes y habilidades necesarias para aumentar su capacidad de funcionamiento afín de lograr condiciones de vida que conduzcan hacia la salud ${ }^{(2,3)}$.

Pero para que estos objetivos se puedan conseguir hay que llegar a toda la población, lo que significa una actuación de alfabetización en salud. Si alfabetización significa la capacidad de entender y expresar correctamente, por medios hablados y escritos, ideas, informaciones y sentimientos, la alfabetización en salud posibilita el desarrollo de habilidades sociales, cognitivas, procedimentales y actitudinales de las personas para acceder, entender y utilizar la información que les permite la promoción y el mantenimiento de una buena salud ${ }^{(4)}$.

La idea de habilidades para la vida definida por la Organización Mundial de la Salud (OMS) y desarrollada en el marco de las Escuelas Promotoras de Salud en Colombia y en la Red de Escuelas Saludables en Europa ${ }^{(5)}$ en la década de los 90, propone el desarrollo de competencias psicosociales e interpersonales que ayuden a las personas a tomar decisiones conscientes, solucionar problemas, pensar críticamente y con creatividad, comunicarse efectivamente, construir relaciones saludables, simpatizar con los demás y manejar sus vidas de una manera sana y productiva ${ }^{(6)}$.

Por todo lo expuesto, la escuela es un escenario perfecto para el desarrollo de prácticas de promoción de la salud ${ }^{(7)}$, ya que, en la infancia y especialmente en la edad escolar es cuando se adquieren y se construyen las capacidades cognitivas y motoras que determinan, en gran parte, las capacidades futuras de ser y estar en el mundo ${ }^{(8)}$. Además, los jóvenes sanos tienen más probabilidades de lograr sus objetivos académicos y sociales y tienen menos probabilidades de emprender conductas de alto riesgo ${ }^{(9)}$.

Más allá de esto, la función política y social de la escuela promueve, a través de los procesos educativos, actividades de naturalezas diversas que, extrapolando el territorio escolar, alcanzan el espacio comunitario y favorecen intensas interacciones y reflexiones sociales sobre la salud, las cuales son necesarias para el desarrollo 
de competencias en salud desde el punto de vista holístico e integral que defendemos.

En Brasil es un desafío trabajar la Promoción de la Salud pues el actual sistema de salud, en la práctica, no tiene en cuenta esta perspectiva amplia que implica la integración intersectorial y la comprensión integral de la salud como un fenómeno social que capacita a los individuos y también a la comunidad ${ }^{(10)}$.

Las investigaciones y evaluaciones específicas sobre la promoción de la salud en la escuela constituye un desafío pues están relacionados con la descripción de los proyectos e intervenciones y/o la formación de los profesionales sanitarios, sin tener en cuenta el trabajo de los maestros en el contexto educativo.

El profesorado es fundamental en el desarrollo de la propuesta de la promoción de la salud en la escuela, pues sirve de modelo para los estudiantes, evalúa los currículos y programas. Además, genera conocimientos, reflexiones y recursos para la inclusión efectiva de la salud en el contexto educativo $^{(11)}$.

La International Union for Health Promotion and Education $^{(9)}$ señaló que los programas de promoción de la salud en las escuelas son más eficaces cuando se guían por temas clave, incluyendo el desarrollo de habilidades y competencias de los maestros a través de su formación y del suministro de recursos con los que puedan replicar en la práctica la formación teórica recibida.

Pero surgieron las siguientes preguntas ¿Cuáles son los afectos del profesorado sobre el ambiente escolar y sobre las acciones promotoras de la salud? ¿Qué aspectos involucran a los maestros en las acciones que promuevan la salud en su centro educativo?

El afecto es un reflejo de las relaciones que surgen en el curso de las historias de las personas y va adquiriendo sentido a lo largo de las mismas ${ }^{(12)}$. Es decir, se forma a partir de la interacción con la realidad, la cual genera emociones afectivas momentáneas, conscientes o inconscientes ${ }^{(13)}$. La construcción de lazos de la persona con el mundo está mediada por el entorno en el que vive, donde la emoción, el lenguaje y el pensamiento llevan a la acción ${ }^{(14)}$. Por lo tanto, somos las actividades que desarrollamos, somos la conciencia que refleja el mundo, y somos los afectos que aman y odian el mundo y con estos nos identificamos con todo que nos rodea ${ }^{(13)}$.

El afecto es visto en este trabajo como una categoría de análisis que " $[\ldots]$ ofrece, además de los conocimientos sobre el medio ambiente, la posibilidad de observar cómo actúan los individuos y se posicionan en su entorno"(10). Resulta de la percepción de un cierto estado del cuerpo que obtiene significado por la consciencia ${ }^{(15)}$. Así, los afectos son emociones que tienen origen en el cuerpo y son percibidos y adquieren sentido por el sujeto. Todo ese proceso constante y dialéctico compone la afectividad.

En el caso del afecto del profesorado, éste se construye a través del proceso dialéctico entre el docente y la escuela. Se puede acceder a él a través de la comprensión de las actitudes y percepciones circulantes en el ámbito educativo. Estos afectos son los que favorecen o no su participación en las acciones que promueven la salud.

En base a todo lo expuesto, el objetivo de esta investigación fue comprender la afectividad y la promoción de la salud en la escuela a través de la construcción de significados por el maestro.

\section{MÉTODOS}

El presente estudio exploratorio y descriptivo se realizó con un enfoque cualitativo que se ajusta al propósito de comprender las percepciones y los sentimientos de los participantes. La metodología utilizada en este estudio tiene como base la propuesta de los mapas afectivos ${ }^{(16)}$ y las entrevistas semiestructuradas. Los mapas afectivos proporcionan la superación de la dualidad entre cognición, afecto y la representación de los espacios, pues en este contexto cualquier ambiente puede ser percibido como un territorio emocional.

La dimensión afectiva facilita la comprensión del territorio existencial de los maestros, pues pueden promover cambios en sus actitudes, en su cuerpo, su salud y su entorno. Estos cambios se basan en una manera de comprender cómo se ven afectados por el ambiente y por las lecturas que hacen de él, de manera que los conducen a adoptar nuevas posturas conscientes de uno mismo y del contexto en el que viven.

Sin embargo, el objetivo del estudio no es establecer una lógica lineal entre el afecto de los maestros con la escuela y su participación en las acciones promotoras de la salud, sino cartografiar sus afectos en el espacio de la educación, e identificar aquellos que interfieren con él y con su participación en las acciones de promoción de la salud.

El estudio se realizó entre los meses de junio de 2010 y enero de 2011 en la Escuela de Aplicación Yolanda Queiroz, en Fortaleza-Ceará-Brasil, una institución cuyas actividades se centran en la educación de los niños en edad preescolar y escolar en situación de vulnerabilidad económica y social y que, además, funciona como un campo de prácticas de los estudiantes de término de grado del Centro de Humanidades y Salud de la Universidad de Fortaleza (UNIFOR).

La opción por esta escuela se debe a que su currículo escolar se enriquece con actividades tales como la informática, las artes plásticas, la psicomotricidad, la danza y la educación física. Además, recibe una gran inversión 
debido al programa de responsabilidad social y extensión de la UNIFOR para el área de la salud pues envía estudiantes de los cursos de grado del área de la salud para realizar sus prácticas. Todo eso coloca esta escuela en lugar de gran influencia en la práctica de los preceptos establecidos por la OMS para una Escuela Promotora de Salud.

Los docentes han sido elegidos como sujetos de la investigación porque su papel es fundamental para conseguir cambios en las rutinas de la escuela en favor de acciones que promueven la salud y porque contribuyen a la superación de las estructuras ideológicas, cuando fomentan la salud y sus prácticas, a través de debates educativos.

Los criterios de inclusión en la investigación fueron: tener por lo menos un año de vínculo de trabajo con la escuela, tener contacto directo con acciones que promueven la salud y mostrar interés y disposición en participar. Del efectivo compuesto de veinticinco maestros, doce cumplieron los requisitos. No obstante, sólo diez participaron de la investigación, pues dos de ellos fueron excluidos debido a encontrarse enfermos y retirados del trabajo.

Los participantes tenían entre 28 y 58 años. El tiempo dedicado a la enseñanza varió entre 6 y 19 años. Todas eran mujeres y la mayoría (seis maestras) enseñaba portugués, matemáticas, ciencias naturales y sociales. Las demás enseñaban informática (una maestra), lectura infantil (una maestra) y bellas artes (dos maestras).

A las maestras les fue solicitado que hiciesen un dibujo que representase su escuela y que iba acompañado de comentarios sobre su significado, sobre los afectos que surgieron a partir de él, la comparación de la escuela con algún objeto, y los espacios de la escuela más utilizados por las participantes. El análisis del dibujo de cada una de las maestras en conjunto con sus contestaciones constituye su mapa afectivo. Así, los mapas se presentan como una construcción que los investigadores elaboran a partir de los datos (dibujo - presentados en el cuadro II - y su significado) aportados por las maestras. Para hacer este análisis se siguió las etapas contenidas en en el cuadro $\mathrm{I}^{(17)}$.

Los mapas en esta investigación han sido adaptados con el fin de entender el afecto existente entre la docente y su ámbito escolar, teniendo en cuenta la investigación original $^{(17)}$ de que estudió los afectos de las personas con respecto a las ciudades. Esta metodología se ha adaptado y utilizado en varios estudios sobre la relación afectiva de los sujetos respecto a entornos como el centro de la ciudad de Fortaleza ${ }^{(18)}$, albergues y ciudad para adolescentes ${ }^{(19,20)}$, percepción de la naturaleza por niños de contextos urbano ${ }^{(21)}$ y hogares de ancianos ${ }^{(22)}$, entre otros.

Se utilizó entrevistas semiestructuradas para mejor comprender la percepción de las maestras sobre las acciones realizadas para la promoción de la salud y sus formas de participación en ellas. Las entrevistas fueran gravadas, transcritas y acontecieron dentro de la propia escuela en salas reservadas. Las preguntas de orientación fueron: las acciones promotoras de salud en las que cada maestra participó, su percepción sobre las mismas, los afectos cuando realizan las acciones sugeridas por la escuela y cómo perciben su participación en estas acciones.

La construcción de los mapas afectivos incluyó los siguientes aspectos y etapas: a) las características sociales de las participantes: edad, sexo, tiempo de trabajo en la escuela, la asignatura que enseña; b) la forma de ver, sentir y representar la escuela, utilizando lápices de colores y tizas de cera; c) las reflexiones sobre la escuela: la expresión oral sobre lo que el sujeto piensa acerca de ella. Este paso permite conocer la elaboración de metáforas y un resumen de la comprensión de la comunicación del afecto con el ambiente de la escuela.

Para el análisis de las entrevistas se ha elegido el análisis temático que destaca dos procesos distintos: la construcción de categorías y la cuantificación de la expresión $^{(23) .}$ Son tres pasos básicos relacionados al análisis temático: pre-análisis que está directamente relacionada con la elección de los documentos a analizar; descripción analítica que son los procesos de codificación, clasificación y categorización; e interpretación de inferencia que consiste en el establecimiento de relaciones entre la realidad que se vive y las personas.

Esta investigación fue sometida al Comité de Ética de la Universidad de Fortaleza y aprobada con el n . 10/319. Para proteger el anonimato de los participantes, sus nombres fueron reemplazados por números que corresponden al orden en el que se llevó a cabo la entrevista. Todos los participantes firmaron el termo de consentimiento libre y esclarecido.

\section{RESULTADOS}

A partir de los datos de los mapas afectivos surgieron diversos y reveladores afectos de las maestras al hablar de su relación con la escuela, entre ellos, amistad, compañerismo y unidad, que se consideran importantes para que la escuela sea vista como un lugar placentero.

La maestra 1 (Cuadro II) expresa la amistad y el compañerismo presente en esta escuela donde cada uno se centra en el objetivo de construir una educación de calidad. Sin embargo, al hablar sobre el dibujo queda claro su aflicción por la necesidad de emplear la mayor parte del tiempo de su vida a la enseñanza, lo que significa un trabajo de mucha dedicación.

La maestra 8 (Cuadro II) expresa en su mapa el gran valor de la escuela en su vida, pues ha donado 19 años de su 
Cuadro I - Etapas del análisis a través de la adaptación del instrumento creado por Bomfim ${ }^{(17)}$.

\begin{tabular}{|ll|}
\hline Identificación & Edad, sexo, tempo de trabajo en la escuela; asignaturas que enseña. \\
Estructura & Los dibujos son clasificados en acuerdo con la aprehensión cognitiva del espacio que expresan \\
analogía, el sentimiento o estado del ánimo de lo participante. \\
$\begin{array}{l}\text { Significado } \\
\text { Cualidad }\end{array}$ & Explicación del participante sobre el dibujo. \\
Sentimiento & Atributos del dibujo y de la escuela apuntados por el participante. \\
Metáfora & Expresión afectiva de lo participante sobre el dibujo y la escuela. \\
& Comparación de la escuela con algo (por lo participante) que tiene por función la elaboración de \\
metáforas.
\end{tabular}

existencia para el trabajo docente en este lugar. Su conexión con la escuela sigue fuerte, en la medida en que la considera como su segundo hogar y la razón de su existencia. Con esta misma visión la maestra 9 (Cuadro II) hizo un dibujo que representa la escuela como su familia, donde hay una gran amistad con sus compañeras de trabajo y donde se desarrollan proyectos integrales siempre en busca de mejoras. Por otro lado, la maestra 10 percibe el ambiente educativo como un barco con bordes irregulares que deben ser arreglados y que fluye hacia la cosecha de buenos frutos, que resultan del sentimiento de armonía y complicidad entre las maestras pero también requieren dedicación y responsabilidad por la protección de los niños y por pasar más tiempo en la escuela que en su casa.

Esta identificación se fundamenta en el diálogo con el entorno y en la construcción del lugar que constituye el proceso de apropiación ${ }^{(24)}$. Proceso en que las personas, individual o colectivamente, identifican sus propios territorios, sus conocimientos, sus relaciones sociales y al mismo tiempo, buscan satisfacer sus necesidades de pertenencia y de identificación ${ }^{(25)}$.

En este proceso no debe estar ausente el afecto, ya que por medio de él las personas atribuyen significado a sus acciones, y la experiencia emocional en un lugar está basada en la relación dialéctica entre el sujeto y el medio ambiente, generando un proceso de transformación mutua.

Por consiguiente, con estas ideas y a modo de ejemplo, constatamos que algunas maestras, al responder a los estímulos del dibujo definen la escuela como una familia, un sol en sus vidas, un lugar de amistad y apoyo, en el que se realiza el trabajo en paz, unión y amor como se percibe en los dibujos y discursos de las maestras 5,7 y 3 (Cuadro II).

El dibujo de la maestra 2 (Cuadro II) muestra la percepción de la escuela como un lugar de amistad, en que la solidaridad y unidad entre las maestras sirven de protección a los niños. Durante la elaboración del dibujo, esta participante expresó su percepción de la escuela como un rompecabezas donde cada pieza está en la responsabilidad de un profesor para contribuir a la protección de los niños y para construir un futuro mejor para ellos.

Sin embargo, las maestras no se reconocen como personas con necesidades de salud y bienestar y con oportunidades para participar en acciones destinadas a promover su salud. No se perciben como protagonistas en la construcción de un ambiente educativo que promueva su propia salud, aunque demuestran una gran consideración por aquellas que se dirigen hacia la mejora de la salud de los niños:

"Las acciones de salud son muy importantes para los padres y alumnos, ya que es una manera de que los estudiantes tomen conciencia de la prevención de enfermedades y aprendan hábitos saludables como es una buena alimentación." (Maestra 1)

"Pienso que es válido, porque los estudiantes de la Universidad traen informaciones que nosotros a veces no tenemos. Cuando viene algo diferente, algo nuevo, los alumnos se muestran más interesados." (Maestra 4)

Esto parece ser debido a la creencia de algunas participantes en que la escuela debe orientarse a la realidad de los niños, que debe servir para transformar el contexto en el cual el estudiante vive y ofrecer un mundo de mejores oportunidades, incluyendo las acciones destinadas a su salud.

Por otra parte, en la escuela investigada, los relatos dan cuenta que las intervenciones destinadas a la salud se llevan a cabo de forma aislada y puntual por los estudiantes de prácticas de los cursos de grado. Ellos no promueven un intercambio de ideas o decisiones que beneficien a la comunidad escolar. La gran mayoría de las acciones ejemplificadas por los profesores son intervenciones realizadas por los estudiantes de prácticas, destinadas a la prevención de enfermedades, tales como teatro informativo para la prevención del dengue, prevención de influenza H1N1 y diagnósticos para la demanda de servicios 
Cuadro II - Presentación de los dibujos de las maestras ( $\mathrm{n}=10)$.

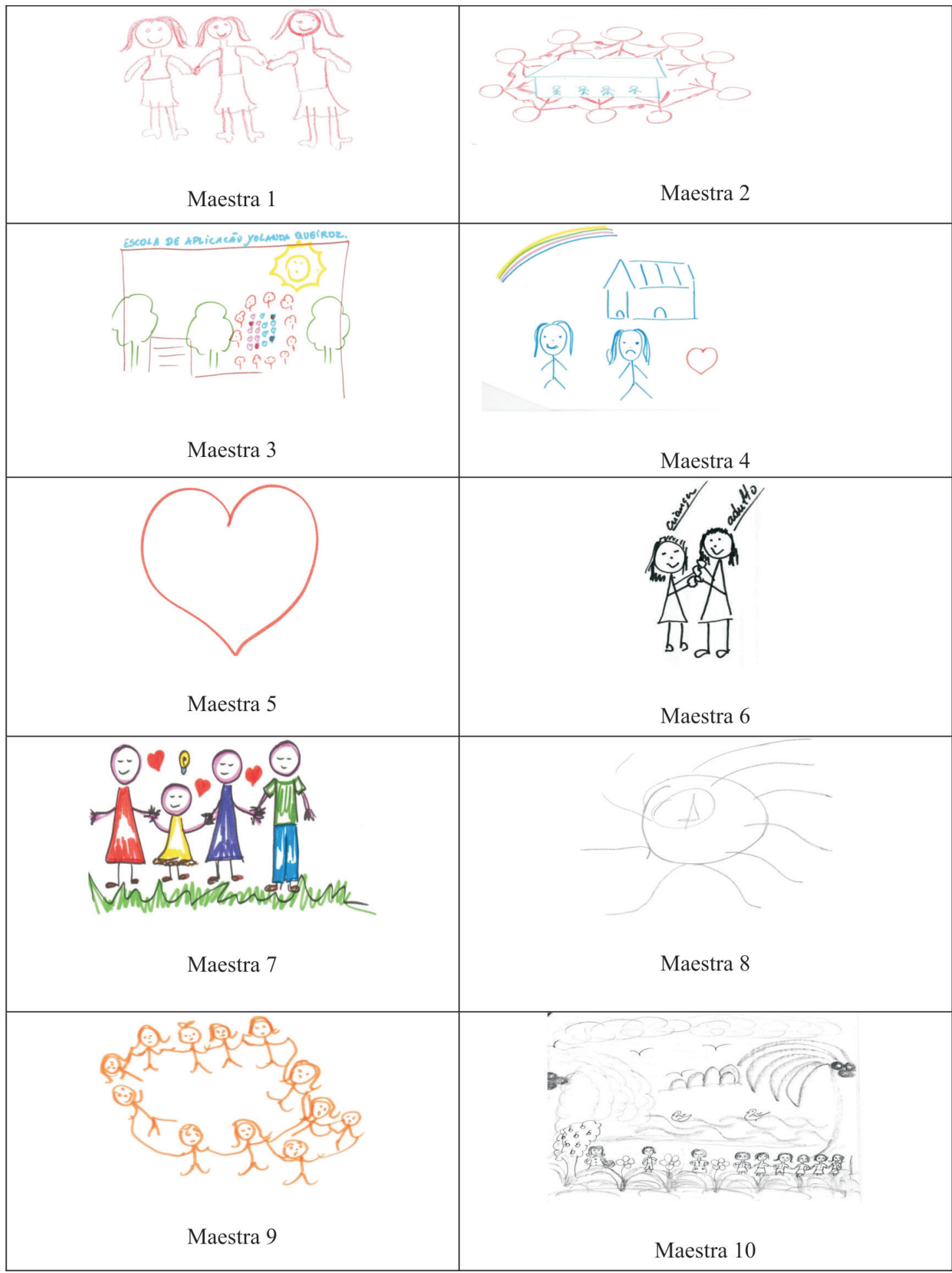

psicológicos. Por parte del profesor de la escuela las acciones para la salud son las normas de higiene personal entre ellos: baño colectivo, cepillarse los dientes, el hábito de lavarse las manos antes de las comidas, higiene de los niños con piojos, etc.

En realidad, no hay por un lado acciones promotoras de salud, y por otro lado acciones preventivas de enfermedades. 
Este es un debate importante en el campo de la salud pública, debido a que varias de las intervenciones preventivas son parte de estrategias más amplias que incluyen la promoción de la salud atendiendo a las demandas específicas de un contexto. Los programas enfocados en un sólo problema, como enfermedades específicas, tienen grandes desventajas, pues ocasionan superposición de acciones y multiplicidad de esfuerzos y recursos ${ }^{(4)}$. Además, no se debe olvidar que las estrategias para la alfabetización para la salud no deben atender exclusivamente a las capacidades individuales de los ciudadanos, sino hay que contextualizarlas en un escenario concreto pues resultan de la interacción entre las demandas de los diferentes sistemas de salud y las habilidades de los individuos inmersos en ellos ${ }^{(6)}$.

Las acciones destinadas a prevenir enfermedades deben ser realizadas de manera participativa, deben estimular la conciencia crítica y crear estrategias de participación comunitaria, no solamente hacia la prevención de enfermedades sino también en la promoción de la salud ${ }^{(26) .}$

No obstante, en la escuela estudiada se muestran evidentes conflictos en la relación del profesorado con los estudiantes en prácticas que realizan acciones de salud en la escuela. La forma como las ejecutan representa el principal problema para la inserción de los docentes, pues no consideran al maestro ni a la comunidad educativa. Las siguientes declaraciones ilustran esta situación:

"Ellos toman los niños y se los llevan, no participamos, somos meros espectadores." (Maestra 10)

"Cuando los aprendices llegan no se coordinan con nosotros, no es un proyecto conjunto con las maestras, es un proyecto de ellos y estamos excluidas." (Maestra 7).

Esto indica un desafío importante para la propuesta de promoción de la salud en la escuela pues el contexto educativo no es percibido como un espacio holístico, donde también deben considerarse los aspectos ambientales, físicos de la área y emocionales de todos. Además hace emerger en los profesores un sentimiento de exclusión y desvaluación.

La participación en los movimientos sociales no es solo un proceso racional y no siempre lleva a un aumento del pensamiento crítico, de la misma forma que el conocimiento de la legislación no conduce, necesariamente, a la participación en este tipo de acción ${ }^{(12)}$. Aun cuando una persona actúe en nombre del bien común, la acción implica el ejercicio de la motivación individual, como una necesidad del ego, del deseo y del afecto que es una dimensión fundamental ${ }^{(10)}$.

La escuela estudiada aparece como un simple receptor de las intervenciones esporádicas de los equipos de salud, externos a la comunidad educativa. Este es definido como un enfoque tradicional pues parte de una comprensión de salud como ausencia de enfermedad y no corresponde al concepto de promoción de la salud, que debería relacionarse con las expectativas y las necesidades del contexto escolar ${ }^{(27) .}$

La International Union for Health Promotion and Education ${ }^{(9)}$ señala que las acciones de educación para la salud en la escuela no deben ser esporádicas, sino constantes, formando parte de un proyecto en el que participen todos los implicados.

La escuela estudiada puede ser percibida como un ambiente posible para el sustento de propuestas de promoción de la salud, en la medida que produce sentimientos placenteros en el profesorado, impulsado por sus afectos. De esta forma las maestras pueden comprometerse con el movimiento para transformar la escuela en un espacio en constante proceso de mejoría.

Elvínculo con los niñosy la percepción de suaprendizaje, el amor a la profesión, el sentido de responsabilidad con el trabajo y la presencia en el aula son factores que ofrecen bienestar a los maestros e implican su consideración y apoyo a las acciones promotoras de salud. Los sentimientos de placer, forman parte de la idea salutogénica, pues llevan a un aumento de las potencialidades para no enfermar, intervienen en las capacidades de superación de las adversidades y generan salud en los espacios donde se vive $^{(28)}$.

Los afectos existentes hacia el ambiente escolar no son suficientes para la implicación de las maestras en las acciones promotoras de la salud por la manera descontextualizada como son realizadas. No hay integración ni participación de las maestras en los objetivos y estrategias para la promoción de la salud en la escuela pues se llevan a cabo sin tenerlas en cuenta. Esto representó el principal obstáculo para la complicidad y colaboración de las maestras en las acciones desarrolladas por los estudiantes de grado de salud. Es necesario reconocer a los maestros como autores fundamentales para la sostenibilidad de las referidas acciones promotoras de la salud. El maestro debe ser visto como un agente facilitador y propiciador del proceso de aprendizaje pues de su relación con los estudiantes como protagonistas de su aprendizaje, se pueden generar nuevo conocimiento y desarrollar habilidades para la vida y competencias de salud ${ }^{(4)}$.

\section{CONSIDERACIONES FINALES}

La investigación ha enseñado que los sentimientos de amistad, compañerismo y unidad del profesorado son importantes para que la escuela sea vista como un lugar placentero. El vínculo con los niños, el amor a la profesión, el sentido de responsabilidad con el trabajo influye en la valoración de las acciones promotoras de salud. 
Pero, los conflictos en la relación del profesorado con los estudiantes en prácticas que realizan acciones de salud en la escuela y la forma como las ejecutan representa el principal problema para la inserción de los docentes.

Así, se hace urgente la necesidad de intervenciones de promoción de la salud en la escuela donde el maestro sea el protagonista y pueda participar en la implementación de estrategias que posibiliten el desarrollo del entorno educativo en relación a este tema.

Por esto proponemos que se amplíe las discusiones sobre la educación y la promoción de la salud en la escuela, teniendo en cuenta el trabajo y los afectos de los maestros en este contexto.

Remarcamos que esta investigación no es concluyente y se necesitan otras investigaciones que le den continuidad y amplitud con el fin de aumentar la comprensión de los elementos que conducen a la construcción y generación de escuelas promotoras de salud.

\section{REFERENCIAS}

1. World Health Organization. The Bangkok Charter for Health Promotion in a globalized world. in: milestones in health promotion statements from global conferences. Geneva: WHO; 2009. p. 24-28.

2. Marinho JCB, Silva JAS. Conceituação da educação em saúde e suas implicações nas práticas escolares. Ensino Saúde Ambiente. 2013;6(3):21-38.

3. Salci AM, Macenno P, Rozza GS. Educação em saúde e suas perspectivas teóricas: algumas reflexões. Texto \& Contexto Enferm. 2013;22(1):224-30

4. Falcón M, Luna A. Alfabetización en salud: concepto y dimensiones. Rev Comunicación y Salud. 2012;2(2):91-8.

5. Camargo-Ramos CM, Pinzón-Villate GY. La promoción de la salud en la primera infancia: evolución del concepto y su aplicación en el contexto internacional y nacional. Rev Fac Med (Bogotá). 2012;60(Supl 1): 62-74.

6. Builes BMM, Arango CPM, Carmona NDM. Otra Mirada a la escuela: transformacion necessária para el siglo XXI. Rev Colombiana Ciencias Sociales. 2015;6(1):136-57.

7. World Health Organization. What is the evidence on school health promotion in improved health or preventing disease and, specifically, what is effectiveness of the health promotion schools approach? [Internet]. Copenhagen; 2006 [citado en 2015 Feb 11].
Disponible en: http://www.euro.who.int/_data/assets/ pdf_file/0007/74653/E88185.pdf_

8. Afonso CMC, Tavares MFL, Luiza VL. Escolas promotoras de saúde na America Latina: uma revisão do período 1996-2009. Rev Bras Promoç Saúde. 2013;26(1):116-26.

9. International Union for Health Promotion and Education. Promover la Salud en la escuela: de la evidencia a la acción. Saint-Denis; 2013. [citado en 2015 Feb 11] Disponible en: http://www.iuhpe. org/images/PUBLICATIONS/THEMATIC/HPS/ Evidence-Action_SP.pdf

10. Falkenberg MB, Mendes TPL, Moraes EP, Souza EM. Educação em saúde e educação na saúde: conceitos e implicações para a saúde coletiva. Ciênc Saúde Coletiva. 2014;19(3):847-52.

11. Silva RD, Catrib AMF, Collares PMC, Cunha SMT. Mais que educar... ações promotoras de saúde e ambientes saudáveis na percepção do professor de escola pública. Rev Bras Promoç Saúde. 2011;24(1):63-72.

12. Sawaia BB, Silva DNH. Pelo reencantamento da Psicologia: uma busca da positividade epistemológica da imaginação e da emoção no desenvolvimento humano. Cad CEDES (UNICAMP).2015;35:343-60.

13. Sawaia BB. Psicologia e desigualdade social: uma reflexão sobre liberdade e transformação social. Psicol Soc. 2009;21(3):364-72.

14. Lane STM. A mediação do emocional na constituição do Psiquismo. In: Lane STM, Sawaia BBS, organizadores. Novas veredas da psicologia social. São Paulo: Brasiliense: EDUC; 1994. p. 55-63.

15. Damásio A. O erro de Descartes: emoção, razão e o cérebro humano. São Paulo: Companhia das Letras; 2012.

16. Bomfim ZAC, Nobre BHL, Ferreira TLM, Araújo LMA, Martins AKS, Alencar HF, et al. Affective maps-validating a dialogue between qualitative and quantitative methods. In: Mira RG, Dumitru A. Urban Sustainability Innovative Spaces, Vulnerabilities and Oportunites. Coruña: Deputación de A Coruña, 2014. p. 131-147.

17. Bomfim ZAC. Cidade e afetividade: estima e construção dos mapas afetivos de Barcelona e de São Paulo. Fortaleza: Edições UFC; 2010.

18. Bertini FMA, Bomfim ZAC. Afetos de idosos em intervenções urbanas no centro de Fortaleza/CE. Rev FSA. 2013;10(2):302-26. 
19. Furlani DD, Bomfim ZAC. Jovens de ambiente rural e urbano e sua relação com projetos de vida. In: Leite JF, Dimenstein M. Psicologia e contextos rurais. Natal: UFRN; 2013. p. 117-42.

20. Bomfim ZAC, Alencar HF, Santos SW, Silveira SS. Estima de lugar e indicadores afetivos: aportes da psicologia ambiental e social para a compreensão da vulnerabilidade social juvenil em Fortaleza. In: Colaço VFR, Cordeiro ACF. Adolescência e juventude: conhecer para proteger. São Paulo: Casa do Psicólogo; 2013. p. 317-41.

21. Campos CB, Santos AC, Sarriera J, Bomfim ZAC Fredizi B. Percepción de la naturaleza por niños de contextos urbano y costero: un análisis de dibujos y palabras clave. Rev Latinoamericana Medicina Conductual. 2014;4(1):3-40.

22. Ponte AQ. Afetividade de idosos de vida religiosa consagrada e a moradia na casa de saúde: projetos de vida e processo de estabilização residência [dissertação]. Fortaleza: Universidade Federal do Ceará; 2010.

23. Minayo MCS. O desafio do conhecimento: pesquisa qualitativa em saúde. São Paulo: Hucitec; 2010.
24. Mourão AT, Cavalcante S. O processo de construção do lugar e da identidade dos moradores de uma cidade reinventada. Estud Psicol (Natal). 2006;11(2):143-51.

25. Pol E. La apropiación del espacio. In: Iñiguez L, Pol E, organizadores. Cognición, representación y apropiación del espacio. Barcelona: Universitat de Barcelona; 1996. p. 1-49.

26. Czeresnia D, Maciel EMGS, Oviedo RAM. Os sentidos da saúde e da doença. Rio de Janeiro: Fiocruz; 2013.

27. Mont'Alverne DGB, Catrib AMF. Promoção da saúde e as escolas: como avançar. Rev Bras Promoç Saúde. 2014;26(3):307-8.

28. Francisco RS, Pilar RV, Carmen MR, Mariano HG. Análisis del modelo salutogénico en españa: aplicación en salud pública e implicaciones para el modelo de activos en salud. Rev Esp Salud Pública. 2011;85(2):129-39.

\section{Endereço para correspondência:}

Maxmiria Holanda Batista

Universidade de Fortaleza - UNIFOR

Avenida Washington Soares, 1321

Bairro: Edson Queiroz CEP: 60811-905 - Fortaleza - CE - Brasil

E-mail:maxmiria@unifor.br 